\title{
Frontières
}

\section{La constitution du sens dans l'interdisciplinarité}

\section{Diane Laflamme}

Volume 22, numéro 1-2, automne-printemps 2009-2010

Résilience et deuil

URI : https://id.erudit.org/iderudit/045019ar

DOI : https://doi.org/10.7202/045019ar

Aller au sommaire du numéro

Éditeur(s)

Université du Québec à Montréal

ISSN

1180-3479 (imprimé)

1916-0976 (numérique)

Découvrir la revue

Citer ce document

Laflamme, D. (2009). La constitution du sens dans l'interdisciplinarité

Frontières, 22(1-2), 3-6. https://doi.org/10.7202/045019ar d'utilisation que vous pouvez consulter en ligne.

https://apropos.erudit.org/fr/usagers/politique-dutilisation/ 


\section{$\begin{array}{llllllllll} & E & D & \text { I } & \text { T } & \text { O } & \text { R } & \text { I } & \text { A } & \text { L }\end{array}$}

\section{LA CONSTITUTION DU SENS DANS L'INTERDISCIPLINARITÉ}

\author{
Diane Laflamme, Ph.D., \\ Rédactrice en chef
}

Faut-il s'étonner que la plupart des auteurs qui contribuent à ce numéro de Frontières fassent référence à la réadaptation plutôt qu'à des enjeux de fin de vie? Les textes qu'ils proposent rapprochent les phénomènes de deuil avec la notion plus récente de résilience. Même si chacun de ces deux concepts déploie sa pertinence à partir de champs disciplinaires et de milieux d'intervention qui peuvent sembler éloignés l'un de l'autre, deuil et résilience nous rassemblent en fait autour d'une même visée, celle de la constitution du sens. Les situations de deuil sont un lourd défi lancé à notre capacité de faire sens. Quant aux situations qui provoquent l'émergence de la résilience, la tentation est souvent grande de les classer dans la catégorie du «hors-sens». On en vient à se demander si l'épreuve du hors-sens ne serait pas l'un des multiples chemins qu'il faut emprunter pour qu'il y ait constitution du sens. Nous nous attarderons d'abord à examiner cette capacité que nous avons de «constituer » du sens; nous tenterons ensuite d'observer comment cette capacité se déploie, en prenant comme exemples quatre situations de deuil abordées précédemment dans Frontières et que vient éclairer une co-construction interdisciplinaire faisant appel au concept de résilience.

\section{LA CONSTITUTION DU SENS}

Le sens de mon expérience, de mon vécu de chaque jour, de quoi est-il fait? La phénoménologie husserlienne nous a appris qu'il faut chercher le sens non pas sous la forme d'un objet mais sous celle - bien plus difficile à observer - de quelque chose qui se «constitue », d'un enchaînement qui se déploie dans un horizon. La notion de «constitution» désigne l'activité du sujet par laquelle un objet ou un vécu est donné à ce sujet et acquiert un sens pour lui. Il y a «constitution, écrit Husserl, au sein de certains enchaînements immanents à la conscience qui comportent une unité évidente» (1950, p. 457).

Se référant à Husserl, le sociologue Niklas Luhmann a repris cette notion de constitution du sens pour proposer la notion de systèmes capables de constituer du sens, nommément les systèmes psychiques et les systèmes sociaux - interactions, organisations, sociétés. (Luhmann, 1995, p. 145-147 et 512.) Les opérations qui permettent aux systèmes psychiques de constituer du sens sont des pensées conscientes qui produisent d'autres pensées conscientes en se connectant l'une à l'autre; les opérations qui permettent aux systèmes sociaux de constituer du sens sont des communications qui produisent d'autres communications en se connectant l'une à l'autre. (Laflamme, 2003, p. 79.)

Le sens n'est donc pas à chercher dans un agencement d'objets bien palpables, mais dans un enchaînement de connections entre des pensées et entre des communications. Or ces opérations que sont les pensées conscientes et les communications sont des événements sans durée, qui disparaissent de mon présent aussitôt qu'ils s'y actualisent. Luhmann décrit ainsi la constitution $\mathrm{du}$ sens comme un processus en quatre temps: actualisation, désactualisation, réactualisation et re-virtualisation ; ce processus s'alimente lui-même, tout en demeurant conditionné par le système qui en est porteur (1995, p. 65, 154).

Ce processus qui permet l'émergence du sens contribue-t-il aussi à l'émergence de la résilience ? Les diverses définitions de la résilience comme processus, que le lecteur retrouvera dans plusieurs des textes de ce numéro, invitent à une réflexion à cet égard. Luhmann n'aborde pas l'hypothèse d'une résilience des systèmes capables de constituer du sens. Il les décrit plutôt comme des systèmes autopoïétiques, une caractéristique de tous les systèmes vivants. Ces systèmes se produisent eux-mêmes et ne peuvent se servir que de leurs propres éléments et opérations pour continuer de constituer leurs éléments et opérations. Lorsque l'autopoïèse cesse, le système perd toute capacité; il meurt.

Pour Luhmann, en tant qu'opérations d'un système psychique ou d'un système social, les pensées et les communications consistent toutes deux à établir une "distinction ", à faire la différence entre ce qui est actuel à un moment précis et l'horizon de possibilités à partir duquel le système indique ce qu'il distingue. C'est en retournant à cet horizon de possibilités que le système procédera à la distinction suivante, à la connexion suivante de façon à continuer de faire sens. Même si ses pensées conscientes (système psychique) ou ses communications (système social) ne 
sont que des événements évanescents, qui ne s'actualisent que pour ensuite se virtualiser, le système capable de produire du sens a une puissante ressource à sa disposition, sa circularité: il peut se donner des attentes en conditionnant les connections entre les événements de sens et ces attentes en viennent ensuite à structurer le processus même qui a suscité leur émergence.

Là où Husserl voyait des enchaînements, Luhmann décrit des distinctions et un processus circulaire de connexion entre ces distinctions. Faire une distinction oblige le système à indiquer ce qu'il retient dans son observation, reléguant du même coup ce qui n'est pas distingué (tout le reste) à l'arrière-plan. Un système dont les opérations sont constitutives de sens est capable de distinguer entre l'actualité de ce qui est distingué et la potentialité de tout ce qui reste, qui n'est pas distingué, qui n'occupe pas l'avant-scène mais qui demeure indissociable de ce qu'indique la distinction, ne serait-ce que parce qu'il s'en démarque. Luhmann constate que chaque fois que nous faisons l'expérience du sens, nous faisons l'expérience d'un donné qui déborde ce qu'il donne: un donné qui se distingue de tout le reste - ce qu'il a exclu - tout en le gardant virtuellement accessible à d'autres opérations de distinction.

On peut rapprocher la position de Luhmann relativement au «surplus » de références rattaché au sens de celle de Jean-Michel Salanskis (1997, p. 417-418), qui parle quant à lui d'un enveloppement du sens. Pour Salanskis, cet enveloppement est l'une des caractéristiques de "l'effet-desens» que le développement de la pensée herméneutique «et plus généralement le mouvement de la pensée moderne» ont permis de dégager: "L'enveloppement du sens est sa structure décisive. Ce qui fait que le sens porte l'existence, la temporalise, qu'il passe et qu'il oriente, c'est qu'il est enveloppé en lui-même, que chaque occurrence de l'adresse porte plus de sens qu'elle n'en signifie. Même si aucune élucidation de l'excès du sens dans son occurrence n'a lieu, cet excès est constitutif du sens comme tel. Une occurrence de sens absolument transparente ne serait pas occurrence de sens, mais exhibition d'objet, présentation pleine.»

C'est en me référant à cette remarque de Salanskis sur «l'exhibition d'objet» que je souhaite introduire la notion d'interdisciplinarité à l'intérieur de la réflexion proposée ici sur la constitution du sens. Chaque discipline procède à une mise en objet selon la perspective qui lui est propre; c'est ce qui fait sa force. Cette délimitation n'est cependant qu'une première étape pour le chercheur qui s'intéresse à la constitution du sens; il voudra interagir avec d'autres disciplines pour faire émerger ce que Duchastel et Laberge (1999, p. 64-68) appellent des «médiations interdisciplinaires ». Ces deux auteurs distinguent quatre dimensions de la médiation interdisciplinaire : la construction de l'objet d'étude, les engagements épistémologiques, les détermina- tions méthodologiques et l'interprétation globale de l'objet. J'y ai déjà fait référence pour montrer comment, depuis sa création, la revue Frontières constitue un tel lieu de co-construction interdisciplinaire, un espace où les chercheurs sont invités à tenter des intersections entre diverses modalités de médiations à l'intérieur de la recherche portant sur la mort et le deuil (Laflamme, 2008, p. 3-6).

Toutes les thématiques abordées par la revue Frontières contiennent des références plus ou moins directes aux situations de deuil. Il arrive aussi que le deuil soit choisi comme thème principal d'un numéro. La dernière fois que cela s'est produit, au printemps 2004, c'est autour des blessures des endeuillés que s'est articulée la démarche de constitution du sens. Les médiations interdisciplinaires tentées par nos auteurs faisaient alors appel aux théories et méthodologies de recherche du travail social, de la psychologie, de la psychiatrie, des sciences infirmières, de l'anthropologie, de la sexologie, des sciences religieuses, de la littérature, du récit de vie et des arts plastiques.

Le mot résilience n'a pas été écrit en 2004. Les observations et les démarches de théorisation de nos auteurs n'en rejoignent pas moins celles proposées six ans plus tard dans ce numéro sur la résilience et le deuil. On peut voir à l'œuvre entre ces deux publications un lent processus de co-construction et de médiations interdisciplinaires exploitant les quatre dimensions identifiées par Duchastel et Laberge (1999). Je signale brièvement quatre situations de deuil discutées dans le numéro portant sur le thème «Deuil, blessure vive» que viennent éclairer les considérations sur la résilience: le deuil alourdi par le contexte d'opprobre social, le deuil des parents lors de la perte d'un très jeune enfant, le travail de deuil à l'occasion de la relation de soins et le deuil vécu à une échelle transgénérationnelle. Réciproquement, les recherches et les interventions présentées sur ces sujets il y a quelques années donnent accès à un riche matériau recueilli auprès des endeuillés, que peuvent encore interroger et mettre à profit les chercheurs et les intervenant qui s'intéressent à la résilience.

\section{LE DEUIL ALOURDI PAR LE CONTEXTE D'OPPROBRE SOCIAL}

C'est une mise en objet faisant appel aux ressources de l'anthropologie, du travail social et de la sexologie qui a contribué à mettre en lumière les caractéristiques des deuils sur lesquels pèse, à divers degrés, un opprobre social. On pense ici à des deuils surexposés, qui font suite à un décès qui a attiré l'attention du public et des médias parce qu'il s'est produit dans un contexte de violence ou, à l'inverse, à des deuils clandestins qui ne peuvent se manifester ouvertement parce que la relation avec la personne décédée n'était pas reconnue officiellement par le 
réseau social (Fréchette dans F. 2004, p. 17-21). Le deuil reste un drame privé à maints égards, mais le réseau social auquel participent les endeuillés ne reste pas neutre lors de l'événement de la perte et il peut contribuer à alourdir la peine plutôt qu'à l'alléger. Lors d'un décès par accident ou lors d'une mort subite, les choix que font les survivants en ce qui a trait aux rites funéraires ont attiré l'attention des chercheurs car ils constituent déjà une entrée dans le processus de deuil (Bouchard et Bélanger dans F. 2004, p. 53-57). On pourrait maintenant se demander si la teneur de ces choix ne témoignerait pas également d'une capacité de résilience.

Certains endeuillés voient le vide se faire autour d'eux; d'autres sont assiégés par une meute de présences non sollicitées qui s'imposent dans leur univers. Divers textes d'analyse ou de témoignage du numéro "Deuil, blessure vive» apportaient un éclairage complémentaire à la thématique des deuils surexposés ou clandestins. On trouve dans le traitement médiatique de la mort de Lady Diana un exemple de deuil surexposé pour les proches, accompagné d'un deuil public par des inconnus qui se déclarent affectés par cette perte avec une intensité telle qu'elle en fait presque des proches de la défunte (Lévy et Toupin dans F. 2004, p. 33-37). Le deuil clandestin peut aussi apparaître à l'occasion du bris d'un lien familial considéré trop intense par les autres membres de la famille, comme l'illustre le témoignage de Forest (dans F. 2004, p. 91-95). Le témoignage et le récit de vie font partie des méthodes de recherche qualitative et Frontières donne accès à un riche matériau à cet égard, qui pourrait alimenter les recherches sur la résilience.

Une situation d'opprobre social peut aussi survenir lors du deuil des familles affligées par un décès survenu en contexte de dépendances: abus de psychotropes, jeu compulsif, dépendance amoureuse, sports extrêmes, toxicomanie, etc. (Suissa et Fréchette dans F. 2004, p. 43-49). L'ambiguïté brouille le processus de deuil, constatent les chercheurs, et l'intervention auprès des endeuillés doit en tenir compte.

Sexualité et mort sont deux sujets sur lesquels pèsent des tabous. La sexologie propose des pistes d'intervention qui tiennent compte du travail de deuil que peut provoquer chez une personne la perte de la fonction sexuelle ou l'apparition de dysfonctions sexuelles (Badeau dans F. 2004, p. 74-81). Cet acte tout simple qui consiste à mettre en mots des pertes que la personne se croit trop souvent tenue de garder cachées permet à la sexologie de poser les jalons d'une démarche de réadaptation qui vise une résolution du deuil, mais qui pourrait utilement être élargie pour y intégrer un apprentissage de la résilience.

Bref, le drame que vivent les endeuillés peut être propulsé malgré eux sur la place publique ou, à l'inverse, ignoré par une collectivité qui se montre sourde à la souffrance que provoquent les deuils dont elle ne veut pas entendre parler et aveugle aux blessures qu'elle ne veut pas voir. L'émergence des propriétés qui caractérisent la résilience s'en trouvera alors diversement affectée; les recherches présentement en cours pour définir le concept de résilience sur les plans familial et communautaire pourraient contribuer à la compréhension de cette dynamique.

\section{LE DEUIL DES PARENTS LORS DE LA PERTE D'UN TRĖS JEUNE ENFANT}

La vie peut-elle encore avoir un sens quand on perd son enfant? Faudra-t-il, lors d'un tel drame, exhorter à la résilience celles et ceux que nous voudrions d'abord et surtout consoler? La chercheure Chantal Verdon a interrogé des couples qui avaient perdu leur bébé pendant la grossesse, lors de l'accouchement ou à peine quelques jours ou quelques semaines après sa naissance. Cette étude descriptive menée auprès de 56 couples visait à identifier les indicateurs de santé susceptibles de renseigner les intervenants sur l'intensité des réactions de deuil et à mieux comprendre l'expérience des parents afin de leur apporter le soutien voulu (Verdon dans F. 2004, p.38-42). Le concept de résilience ne faisait pas partie du cadre théorique de l'étude, plutôt axé sur les études de I. Wheeler sur le sens de la perte. Les instruments de mesure comportaient des questions posées aux parents sur leur recherche du sens de la vie, alors défini en référence aux travaux de A. Lang comme "la tendance de l'être humain à recadrer et mettre en perspective les répercussions d'une situation difficile, en changeant au niveau cognitif et émotionnel la façon de voir la situation, et conséquemment de trouver un but et un nouveau sens à l'existence (Verdon dans F. 2004, p. 40). La démarche de recadrage ainsi décrite apparaît très proche des apprentissages que veut susciter l'intervention visant à encourager l'émergence de comportements résilients.

Les sciences infirmières sont un lieu particulièrement fécond de recherche où les besoins de la personne, du couple et de la famille qui viennent de perdre un enfant sont au centre des préoccupations. Fréchette-Piperni (dans F. 2004, p. 58-63) examine un riche matériel recueilli lors du suivi de deuil proposé depuis le début des années 1990 au Centre hospitalier Pierre-Boucher, à Longueuil: plus de 450 familles avaient alors eu accès à cet accompagnement offert par l'équipe des infirmières du Service de natalité. L'un des constats qui saura intéresser les chercheurs dont les travaux portent sur la résilience, c'est que le besoin d'accompagnement peut encore se manifester plusieurs mois, voire des années après la perte.

De précieux témoignages à cet égard ont aussi été recueillis lors d'entrevues menées auprès des membres d'une famille de dix-sept enfants, dont six sont décédés peu après la naissance ou en bas âge (Robitaille dans F. 2004, p. 9-16). Tant la profondeur des blessures jamais entièrement cicatrisées provoquées par la perte que l'importance de la fonction assumée par les tuteurs de résilience - même si cette notion n'est pas explicitement mentionnée lors des entrevues - trouvent une illustration éloquente dans le récit de vie de cette famille.

\section{LE TRAVAIL DE DEUIL À L'OCCASION DE LA RELATION DE SOINS}

D'autres blessures liées au deuil ont pu être identifiées grâce à des psychologues qui ont choisi de porter leur attention sur le vécu des soignants et des accompagnants en fin de vie. Pozo (dans F. 2004, p. 22-27) fournit des données sur le deuil dans lequel s'engage, même avant l'événement du décès, le soignant qui s'occupe d'un parent âgé qui souffre de démence. De Montigny (dans F. 2004, p. 70-73) signale l'existence de ce que l'on pourrait considérer comme une stratégie inédite menant à la résilience: le don de soi dans l'accompagnement d'autrui en fin de vie, comme exorcisme après le décès d'un proche. Le choix d'agir en aidant les autres plutôt que de supporter passivement la perte permettrait de surmonter le sentiment d'impuissance en le troquant contre un sentiment d'utilité auprès d'étrangers qui sont menacés à leur tour par une perte grave.

\section{LE DEUIL VÉCU À UNE ÉCHELLE TRANSGÉNÉRATIONNELLE}

Les recherches et la pratique d'intervention auprès de jeunes délinquants issus de familles émigrées en France ont permis de mettre en lumière un aspect peu connu du phénomène du deuil: ses répercussions à l'échelle transgénérationnelle (De la Vaissière dans F. 2004, p. 50-52). La question identitaire étant le plus souvent abordée en faisant référence au vécu individuel de la personne plutôt qu'à son insertion dans un territoire, on passe souvent sans la voir à côté de la souffrance que comporte la réinvention de soi en contexte migratoire. 
On peut découvrir des résonances entre ces résultats de recherche et la quête de soi que décrit, en mots et en images, l'artiste québécois René Derouin, qui parle de migration pour désigner le parcours qui a fait de lui un artiste ancré dans le territoire des Amériques (dans F. 2004, p. 6-8). Ses milliers de statuettes façonnées dans la terre du Québec et du Mexique représentent des migrants qui avancent en portant diverses charges sur leurs épaules. Il les a fait voyager du nord au sud; il leur a donné une trajectoire qui nous a permis de les voir sortir des musées pour se rendre sur la place publique, en Amérique et en Europe; il nous explique aussi comment il en est venu à les larguer dans le fleuve Saint-Laurent autour des lieux qu'ont habité ses ancêtres. Ce récit de vie et la gestuelle que reproduisent inlassablement les milliers de statuettes qui constituent cette œuvre nous rappellent que la capacité qui est sollicitée par le deuil, c'est celle de porter: porter ses morts en terre, «porter le deuil », avancer en portant quelque chose qui nous écrase sous son poids (Laflamme dans F., 2004, p. 3,5$)$. Il se peut que la capacité de résilience soit aussi une capacité de porter.

C'est également à une échelle transgénérationnelle que se révèle pleinement l'impact des récits autobiographiques que nous ont laissés plusieurs grands auteurs à la suite du décès d'une mère, d'un père ou d'un grand ami. L'œuvre littéraire (Reboul dans F. 2004, p. 28-32) nous fait partager un deuil qui s'inscrit dans une histoire éminemment personnelle, mais où la force de la narration provoque le partage du sens malgré le fossé des générations et l'absence de lien entre les généalogies. Tous ces parcours à travers le temps et les territoires fournissent maints indicateurs à l'observateur qui s'intéresse à la résilience en tant que trait de personnalité.

Ces quatre situations de deuil tirées du numéro de Frontières «Deuil, blessure vive», pourraient se prêter à diverses analyses susceptibles de fournir des données utiles en vue d'une meilleure compréhension des phénomènes de résilience. Dans le texte qu'il a rédigé pour le présent numéro, Michel Hanus propose en

quelque sorte un pont avec ce thème de la blessure lorsqu'il écrit que «c'est dans le vécu des sentiments que se situe la plus grande différence entre le deuil et la résilience». Il y a deuil là où la personne a d'abord vécu un attachement... et pour un être humain il est dans l'ordre des choses de vivre des attachements. Par ailleurs, on souhaite qu'il y ait résilience lors d'un traumatisme qui frappe à l'improviste ou d'une épreuve qui n'est pas le lot de chacun et qui implique souvent une maltraitance de la part d'autrui. Selon Hanus, le deuil et la résilience présentent ainsi «des analogies qui ne sont pas des similitudes».

Michel Hanus a éminemment contribué à éclairer les particularités des deuils complexes; son travail clinique auprès des endeuillés et ses nombreux écrits sur le deuil ont alimenté tant la réflexion des chercheurs que celle des intervenants, en Europe et au Québec. Son décès, survenu en avril 2010, prive les études sur la mort d'une contribution que tous auraient souhaité voir se prolonger et à laquelle nous souhaitons rendre hommage.

Deuil et résilience sont des concepts apparentés, mais ils ne sont pas des synonymes. On pourrait dire que deuil et résilience sont des notions qui se prêtent à des "surimpressions", pour reprendre le titre qu'a donné la photographe Myriam Gaumond à la série de portraits dont sont tirées plusieurs des images présentées dans ce numéro. De telles surimpressions d'images ou de concepts ont une étonnante capacité de provoquer des "effets de sens», pour reprendre l'expression de Salanskis, et d'élargir l'horizon où se font les opérations de connexions entre les pensées et entre les communications décrites par Luhmann. Le travail d'interdisciplinarité est un travail de la pensée et de la communication, dans des systèmes psychiques et des systèmes sociaux capables de constituer du sens dans un monde complexe. Ce monde où nous vivons au quotidien se prête à la réinvention de soi précisément parce que sa complexité défie constamment nos constructions de sens.

Celles et ceux qui ont été blessés par la perte d'un être cher le savent trop bien: trouver un sens à la perte est un travail sans cesse à recommencer, une connexion fragile qui risque de s'arrêter là, confrontée à un vide immense, faute de trouver d'autres connexions qui permettraient de continuer de faire sens. Il en est de même pour la résilience; les textes de ce numéro le répètent maintes fois : son émergence n'est pas un acquis. Tout comme le sens, elle se conquiert de haute lutte.

\section{Bibliographie}

DUCHASTEL, J. et D. LABERGE (1999). "La recherche comme espace de médiation interdisciplinaire", dans Sociologie et sociétés, vol. XXXI, $n^{\circ} 1$, printemps, p. 63-76. FRONTIÈRES (2004). « Deuil, blessure vive », vol. $16, \mathrm{n}^{\circ} 2$, printemps.

HUSSERL, E. (1950). Idées directrices pour une phénoménologie, trad. P. Ricoeur, Paris, Gallimard.

LAFLAMME, D. (2003). "Faire sens grâce à nos opérations conscientes et à nos communications. La notion de sens chez Niklas Luhmann », Frontières, vol. 15, n 2 , p. 78-84.

LAFLAMME, D. (2008). «Médiations interdisciplinaires en études sur la mort », Frontières, vol. 20, n 2, p. 3-6.

LUHMANN, N. (1995). Social Systems, Stanford, Stanford University Press.

SALANSKIS, J.-M. (1997). "Herméneutique et philosophie du sens », dans J.-M. Salanskis, F. Rastier et R. Scheps, Herméneutique: textes, sciences, Cerisy, Paris, Presses universitaires de France. 